\title{
A Brief Algorithm of the Medel Cochlear Implant Fitting
}

\author{
Petrov SM* \\ Department of Otolaryngology, St Petersburg Russia
}

*Corresponding author: Department of Otolaryngology, Petrov SM, St Petersburg, Russia

\begin{abstract}
This article presents a brief algorithm of a cochlear implant fitting from the first fitting to the end of the first session of the implant setting. Most of the articles in the bibliography were written specifically for the future "Guidelines for cochlear implant setup". Links to the world literature are in the references to the above-mentioned articles.
\end{abstract}

\section{Procedure}

a. Switch on the "Maestro" program and connect the processor to the DIB (Medel).

b. Fill the patient data, then place an antenna on the patient's head, read the parameters of the implant [1].

c. Save the patient data and the implant parameters. Carry on telemetry and record its results in a speech processor.

d. Select the frequency range $(250-6500 \mathrm{~Hz})$ [2], set an activation level (95\%), a coding strategy (FS4). The parameters of electrical stimuli should be set to the following: duration $300 \mathrm{~ms}$, interval between stimuli - 600ms [1].

e. Proceed with sequential stimulation of all channels at small electrical levels in the SWEEP mode [1] with an observation of the patient's response. A gradual increase of stimulation levels with three steps each, SWEEP stimulation and save the sequential programs. Step is $0.2-0.3 \mathrm{~dB}$.

f. If the patient's response is detected, the first fitting will end. Afterwards create a map of three steps above the one where the response was detected. Create a configuration with these 4 maps. The fourth map is the last created map. 3, 2 and 1 maps are less in increments of 3 steps. We instruct to switch programs till the setting up the next day. We give our "Instruction" [3] in case of its absence.

g. In the following days, continue parallel increase of the levels of stimuli along all channels with SWEEP stimulation. Monitor the reaction of the patient and take into account the comments of parents and teachers. Remember the intraoperative levels of the stapedial reflex. h. Train Categorical Loudness Scaling (CLS) in the categories "Hear" - "No sound" [4].

i. Relaxometry is performed when approaching the levels of the intraoperative reflex. N.B. Intraoperative - visual - threshold levels of stapedial reflex can be higher than real ones obtained on the impedance meter during the fitting, therefore it is impossible to fully rely on intraoperative threshold levels. We observe reactions of a child and take into account the comments of parents and teachers.

j. Register threshold reflex levels using the SWEEP stimulation-registration program and use them to set C-levels [5]. Based on the results of reflexometry and C-levels of the work program chosen by the parents, create a map which will be the second in the configuration and from that create one program lower by 3 steps (first map) and two program of increasing levels (3-rd and 4-th maps). The electrical threshold levels are set at $20 \mathrm{~dB}$ below the MCL-10\% of the MCL.

k. Train CLS in the categories "No sound", "Soft", "Good", "Loud" [4].

l. Parents and teachers choose a work program.

m. Using the SHCHUP program, determine the maximum comfortable sound pressure level of step noises [6]. The CLS experience helps the child. According to the results of the SHCHUP, adjust the current C-levels through the channels. After that configuration with four maps can be created - the second map is in accordance with the results of the SHCHUP, considering the C-levels of the comfort program chosen by the parents. 
n. In order to choose a work program, we give an instructionexplanation to the parents.

"As you yourself understand, we all go on the same program. Sometimes we hear loudly. But we do not use ear plugs around the life. Why can't an implanted patient hear loudly sometimes? Can. And he should. Sometimes! Therefore, the program at which the patient sometimes hear loudly is optimal- working one" [3].

o. During the session, use MIMIC program to demonstrate to the parents vootiue (on their own ear) the problems of $\mathrm{CI}$ patients and explain to them some features of the auditory perception of their children $[7,8]$.

p. As the last step, carry out a telemetry, open the programs in turn, and agree to the possible proposal of "Maestro" to replace telemetry. Then write the new values of resistance of the electrodes in 4 programs. Afterwards record these programs with the latest telemetry, without changing the C-levels of the last configuration maps.

q. The first fitting session is completed.

\section{References}

1. Petrov SM (2018) First days of the cochlear implant fitting: from the first fitting to the impedancemetry. Austin Med Sci 3(2): 1-4.

2. Petrov SM (2017) Modeling of cochlear implants with different frequency ranges by means of spectrally deprived speech. Journal of Otolaryngology ENT Research 6(4): 1-3.

3. Petrov SM, Tsjuk AA (2015) Instruction for audiologists and cochlear implanted patients. p. 52.

4. Petrov SM (2019) Categorical Loudness Scaling in the Fitting of Cochlear Implanted Children. Scholarly J Otolaryngology 3(1): 218-221.

5. Petrov SM (2017) Practical implementation of the Sweep-session of stimulation-registration in CI fitting. Advanced Treatments in ENT Disorders 1: 13-15.

6. Petrov SM, Gritsjuk MI (2016) Cochlear Implant Fitting in Child: Sweep and Shchup. Austin J Otolaryngol 3(4): 1084.

7. Petrov SM, Pisareva NY (2011) Comb filtered speech as a tool to demonstrate difficulties of speech perception and the importance of auditory training in cochlear implant users. Cochlear Implants International 12(1): 48-52.

8. Petrov SM (2018) For professionals working on the topic of cochlear implantation: Opinions of readers of "Instruction" and participants of Mimic. Adv Treat ENT Disord 2: 1-5.

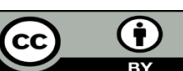

This work is licensed under Creative Commons Attribution 4.0 License

To Submit Your Article Click Here:

Submit Article

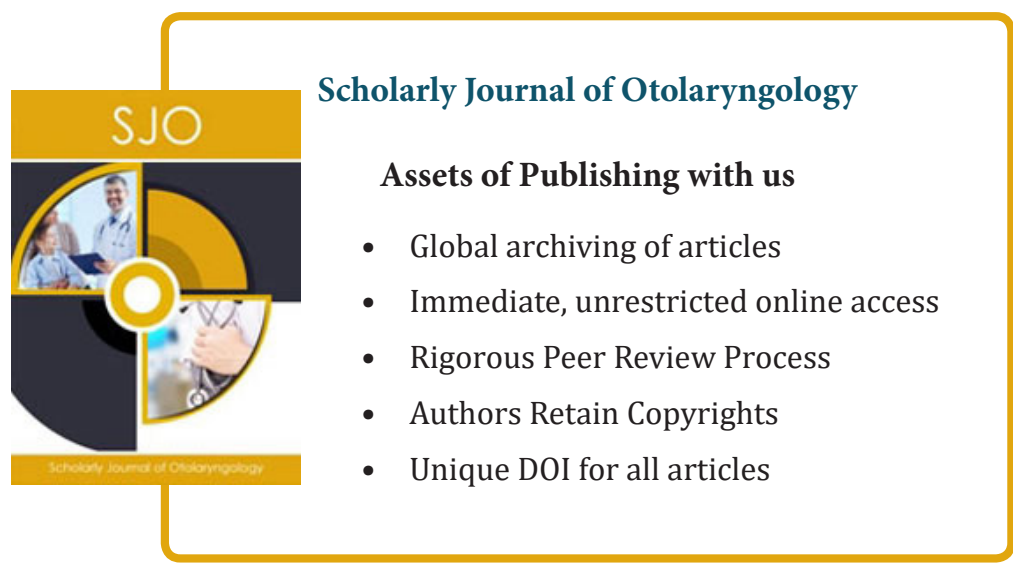

